Case Report:

\title{
Translated Article: Resection of a Rare Proximal Radius Malignant Chondrosarcoma (Grade III) Bone Tumor and Reconstruction of the Forearm Functionality: A Case Report
}

\author{
Sayed Abdulla Jami ${ }^{1}$ (D, Shi Jiandang ${ }^{1 *}$ (D)
}

1. Department of Spinal Surgery, General Hospital of Ningxia Medical University, Ningxia Medical University, Yinchuan, Ningxia, China.

\begin{tabular}{|l|l|}
$\begin{array}{c}\text { Use vour devic to scan } \\
\text { and read thearticle online }\end{array}$ & $\begin{array}{l}\text { Cftat/on Jami SA, Jiandang S. Translated Article: Resection of a Rare Proximal Radius Malignant Chondrosarcoma (Grade } \\
\text { III) Bone Tumor and Reconstruction of the Forearm Functionality: A Case Report. Journal of Research in Orthopedic Science. }\end{array}$ \\
2020; 7(2):79-86. http://dx.doi.org/10.32598/JROSJ.7.2.674.1
\end{tabular}

(c) (i) (8)

Article info:

Received: 14 Aug 2019

Revised: 20 Apr 2020

Accepted: 27 Apr 2020

Available Online: 01 May 2020

Keywords:

Resection, Reconstruction,

Rare proximal, Radius,

Malignant, Chondrosarcoma

\begin{abstract}
A B S T RA C T
Background: Chondrosarcoma is one of the common malignant bone tumors and is characterized by thin tumors. The cells produce tumor cartilage. It occur usually $3^{\text {rd }}$ decade of life, affected more men than women.

Objectives: To perform alternate surgical procedures for remove tumor and obtain fully forearm functionality by reconstruction.

Methods: A patient affected by a rare proximal radius chondrosarcoma bone tumor and it became malignant (grade III). Resection the tumor along with chemotherapy and radiotherapy was done. Surgical treatments are mainly recommended for most types of chondrosarcoma treatment. However, the treatment of chondrosarcoma clinically and surgically is controversial due to different techniques.

Results: Patients was fully recovered with excellent wrist and elbow joint functionality. Reconstruction of bones were fused by the support of metallic implants. There was no tumor recurrence occur during post-operative follow-up period.

Conclusion: Chondrosarcoma of bone generally has an excellent prognosis when optimal diagnosed and treated by an experienced team of specialists. Its clinical treatment is always challenging due to of the rarity of these lesions and few institutions having enough patients to study about it.
\end{abstract}

Note: It was previously published in Chinese in the Orthopedics Journal (ISSN: 1674-8573):

Jinwen H, Jiandang S, Ningkui N, Zongqiang Y, Longyun W, Huiqiang D. [Reconstruction of forearm support after resection of malignant tumors of proximal radius by butt fusion of distal ulna and radius (with a case report and literature review) (Chinese)]. Orthopedics. 2018; 9(4):332-6. [DOI:10.3969/j. issn.1674-8573.2018.04.017]

An erratum article has been published [DOI:10.32598/JROSJ.7.4.674.1] to explain it as the secondary English version of the primary one, based on the 3 rd article of "acceptable secondary publication" of ICMJE's guideline overlapping publication:

International Committee of Medical Journal Editors (ICMJE). 2020. Available from: http://wwwicmje.org/recommendations/browse/publishing-andeditorial-issues/overlapping-publications.html

* Corresponding Author:

Shi Jiandang, MD.

Address: Department of Spinal Surgery, General Hospital of Ningxia Medical University, Ningxia Medical University, Yinchuan, Ningxia, China.

Phone: +86 (137) 09512318

E-mail: shi_jiandang@outlook.com 


\section{Introduction}

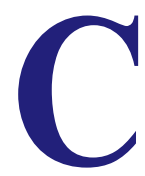

artilage tumor can occur at any age but is more common in adult men [1]. Chondrosarcoma in the proximal middle part of the radius is rare. Also, after extensive radius resection, forearm function reconstruction methods are rarely reported. Postoperative bone defects often require reconstruction of tumor prosthesis or bone graft [2]. Our department of spine orthopedics admitted a patient with chondrosarcoma of the proximal radius bone. After the general resection, the distal ulna and the radius were used for the forearm support skeleton. After 2 years follow-up, the bones of the reconstructed site healed well, and the function of the forearm restored, with no tumor recurrence.

\section{Case Presentation}

\section{Clinical information}

\section{First: general information}

A 36-year-old male patient, due to the right forearm pain for 21 months, admitted to the hospital. The patient had no obvious cause of proximal right forearm swelling since 21 months ago. It was sore and uncomfortable, and the pain was aggravated during exercise. Two months ago, the patient complained of the right elbow joint pain. A mass can be felt on the outside of the joint by touch, gradually increasing in size and aggravating pain. Local hospital x-ray showed cystic expansive bone destruction in the proximal radius. The pain got more serious later, and he visited our hospital.

\section{Second: preoperative examination}

On physical examination of the right swollen forearm on the anterior lateral side and the local part a $3 \times 4 \mathrm{~cm}$ hard mass, with unclear boundary, poor mobility, and local tenderness was visible. Sputum pain was positive. Right upper extremity limb distal blood supply and skin acupuncture were normal. Right elbow flexion of the joint was slightly restricted, but the extension, pronation, and supination of the arm were normal.

Laboratory examination results were blood routine as white blood cell count, $4.86 \times 109 / \mathrm{L}$; medium granulocyte count, $0.06 \times 109 / \mathrm{L}$; and the platelet count, $223.0 \times 109 / \mathrm{L}$. Protein content was $156.0 \mathrm{~g} / \mathrm{L}$ and 2 biochemical complete set: Erythrocyte Sedimentation Rate (ESR), C-Reactive Protein (CRP) and procalcitonin were normal. Tumor marker examination of prostatespecific antigen, carbohydrate antigen CA19-9, CA12-5, and $\alpha$-fetoprotein was normal.

On imaging examination, right ulnar radius and lateral side $\mathrm{x}$-ray showed right radius cystic distension of bone in the proximal midsection, bone destruction, almost involving the full length of the radius, considering the bony exposure to disease. $\mathrm{CT}$ and $3 \mathrm{D}$ reconstruction of the right ulnar radius showed cortical thickening and bone density of the medullary cavity was abnormal, having a long-range of lesions, considering the possibility of osteomyelitis. The right elbow joint MRI scan showed the disappearance of the normal shape of the upper radius and there was an enlargement. Abnormal signals in the bone marrow cavity with edema and thickening of the surrounding periosteum and right radius adjacent, near soft tissue swelling, should be differentiated from eosinophilic granuloma and Ewing's sarcoma. The whole-body CT scan showed abnormal nuclide concentration in the proximal and middle portion of the radius, considering tumor lesions.

\section{Third: pathological examination and diagnosis}

After admission, a CT-guided core trocar puncture biopsy was performed. A grey and white bone tissue with a volume of $0.3 \times 0.3 \times 0.3 \mathrm{~cm}$ was observed by the naked eye. The pathological report was a homogeneous powder stain with cartilaginous visible in the right radius. Cells were considered chondrosarcoma.

Fourth: surgical treatment and reconstruction of forearm support function

Under general anesthesia, the right proximal radius bone tumor was extensively excised and the ulna was far away. Internal fixation was done with titanium plate screws for segmental osteotomy, proximal ulna, and distal radius. An intraoperative right radial bone tumor is cantered, and a posterolateral longitudinal incision was made. The exposure included the proximal radius of soft tissue tumor mass free from the tumor boundary (Figure 1). According to the preoperative MRI intramedullary tumor invasion range (the tumor length is $15 \mathrm{~cm}$ ), the small radius head was exposed to a distance of 18 $\mathrm{cm}$, which is $3 \mathrm{~cm}$ outside the tumor size. We cut the radius internally and removed the proximal and middle portion segment of the radius containing the tumor about $3.6 \mathrm{~cm}$. After the operation, the bone tissue was removed and sent to the pathology examination. 

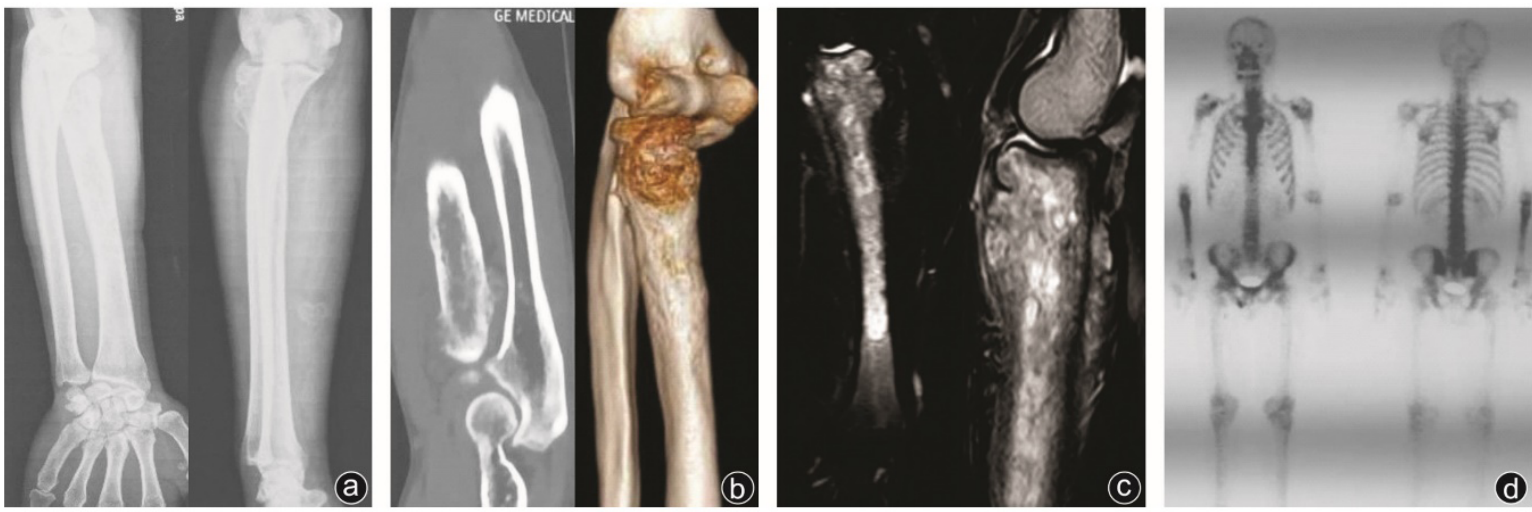

Orthopedic Science

Figure 1. Preoperative imaging data

a: X-ray showed that the proximal and middle end of the right radius was visible, with changes of faecal expansive bone destruction, thickened bone, and the bone was worm-like change; b: CT+ three-dimensional reconstruction showed the radial bone Cortical thickening, abnormal bone marrow density, abnormal calcification; c: The MRI showed obvious enlargement of the upper part of the radius segment, abnormal bone marrow signal, periosteal edema, thickening, swelling of adjacent soft tissue; d: Whole body Bone tomography scan shows concentration of nuclides in the proximal and middle part of radius
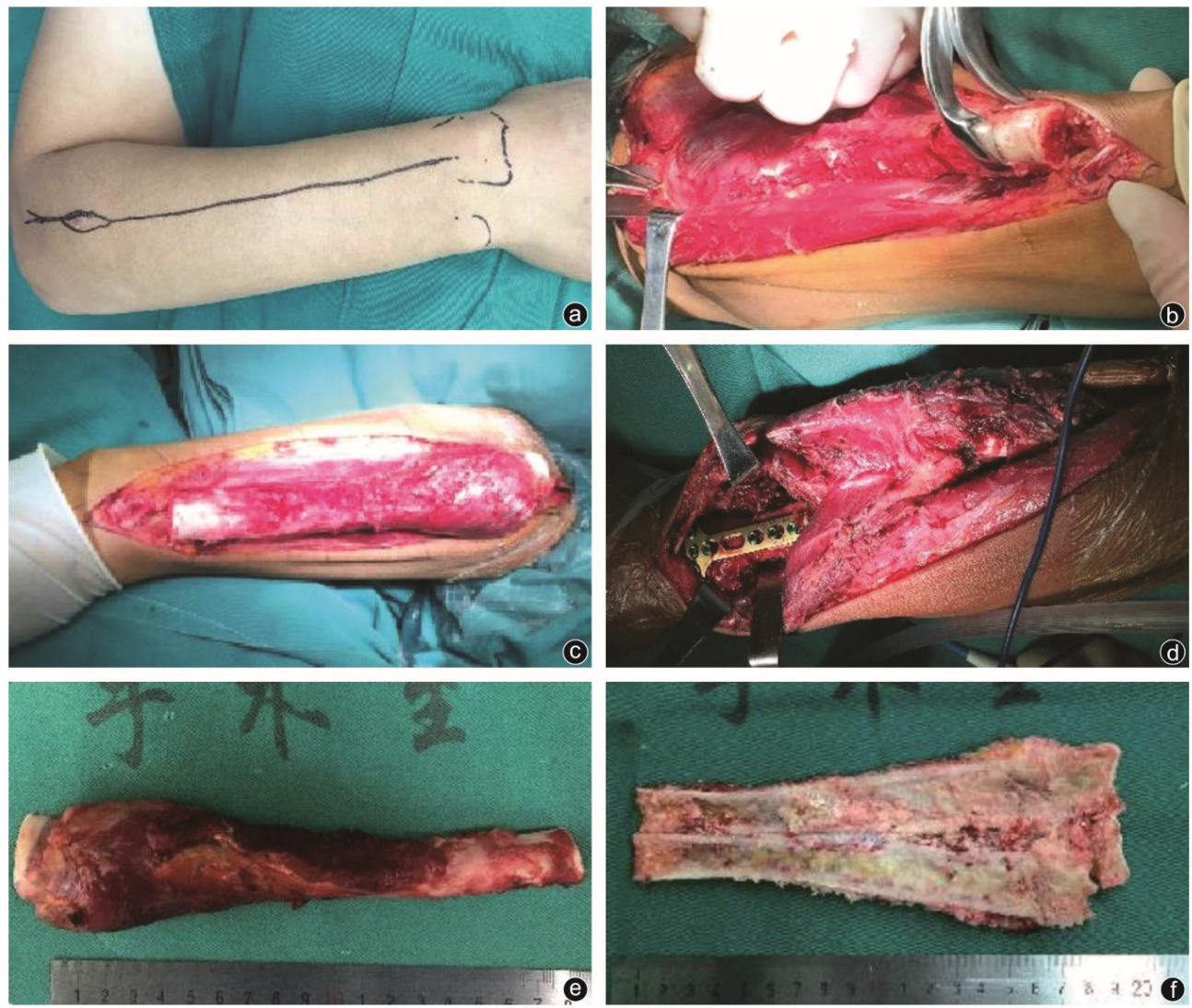

Orthopedic Science

Figure 2. Surgical picture

a: The appearance of the incision and lesion location; $b$ : The radius was exposed at a distance of $18 \mathrm{~cm}$ from the radial head, and the radius was cut within $3 \mathrm{~cm}$ outside of the tumor; c: Remove the proximal middle radius containing where was swelling; $\mathrm{d}$ : revealing the ulna and cutting the ulna at the level of the radial osteotomy, docking the proximal ulnar end with the remaining radial fracture end, titanium plate, screw fixation, reconstruction of the forearm support skeleton; e, f: Resection, Post-tumor tissue appearance and dissection 

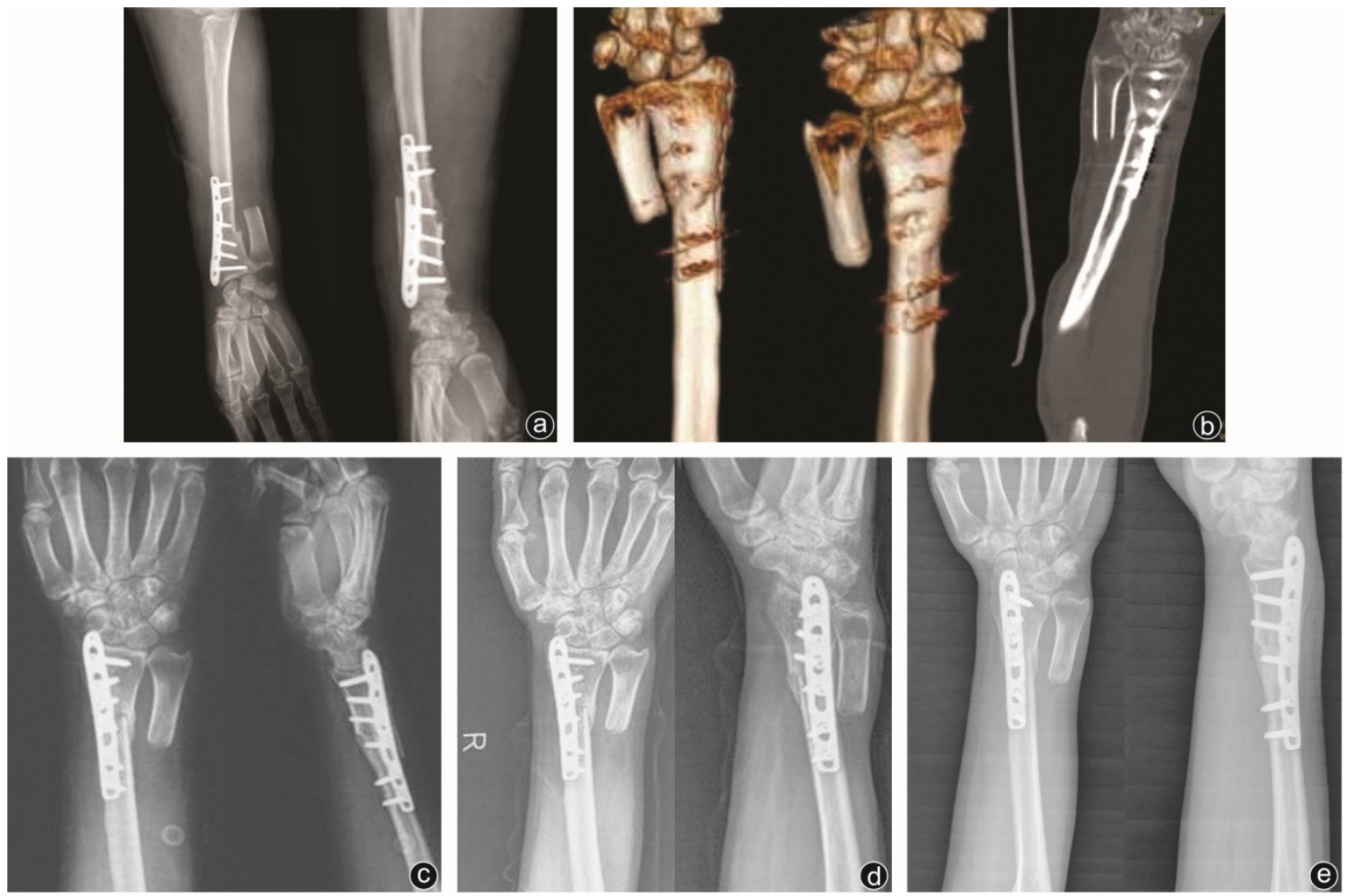

Orthopedic Science

Figure 3. Postoperative imaging data

a: Immediately after the operation, the X-ray film showed good alignment of the ulna and the radial end of the bone; $b$ : CT+3D reconstruction showed bone graft reconstruction after 3 months; c: X-ray film showed 3 months after operation Reconstruction of bon fused and healed well, no tumor recurrence was observed; $\mathrm{d}: 9$ months after operation, the reconstructed bone end healed firmly; e: 2 years after surgery, the reconstructed bone end was completely fused, no elbow joint, wrist dislocation, no tumor relapse

\section{Reconstruction of forearm support function}

The wound was rinsed with distilled water. The instruments and gloves were replaced. The ulna was exposed and truncated at the level of the radial osteotomy, then it was closed and the radial fracture ends were remnant. The wrist joint was slightly rotated, and the titanium plate screw was satisfactory fit after the alignment and joint was fixed. The attention was paid to the pressure at the broken end, and the forearm support skeleton was reconstructed. Finally, the incision was closed. The forearm sling was used to fix the affected location in the functional position (Figure 2).

\section{Fifth: postoperative pathological examination}

Bone tissue removed from the radius had a length of $18 \mathrm{~cm}$ and a diameter of $4 \mathrm{~cm}$. The greyish and whitish tumor tissue can be seen in the medullary cavity with a length of $15 \mathrm{~cm}$. The tumor tissue quality was plastic brittle; the naked eye could inspect the soft tissue around the bone. Microscopically, the mass was composed of chondrocytes and cartilage matrix. It is lobulated and invasive. Pathological diagnosis was right radial chondrosarcoma (grade III); no tumor tissue was seen at the margin.

Six: postoperative radiotherapy and chemotherapy and follow-up

The patient's postoperative pain was relieved. The length of both limbs was equal, and the forearm shape was normal. One month after surgery, three courses of pirarubicin, ifosfamide, recombinant human vascular endothelial inhibition chemotherapy were performed. Also radiation therapy was performed at the same time with the proximal boundary of the level of the olecranon, and the distal boundary of the distal radius, the medial border is the ulnar cortex and the lateral border was the muscle layer.

The prescription dose of 95\% PTV 64 Gy/2 Gy/32 f, 32 times radiotherapy was completed. The fourth cycle of chemotherapy was performed 3 months after surgery. Postoperative $\mathrm{x}$-ray films showed bone grafting fused 

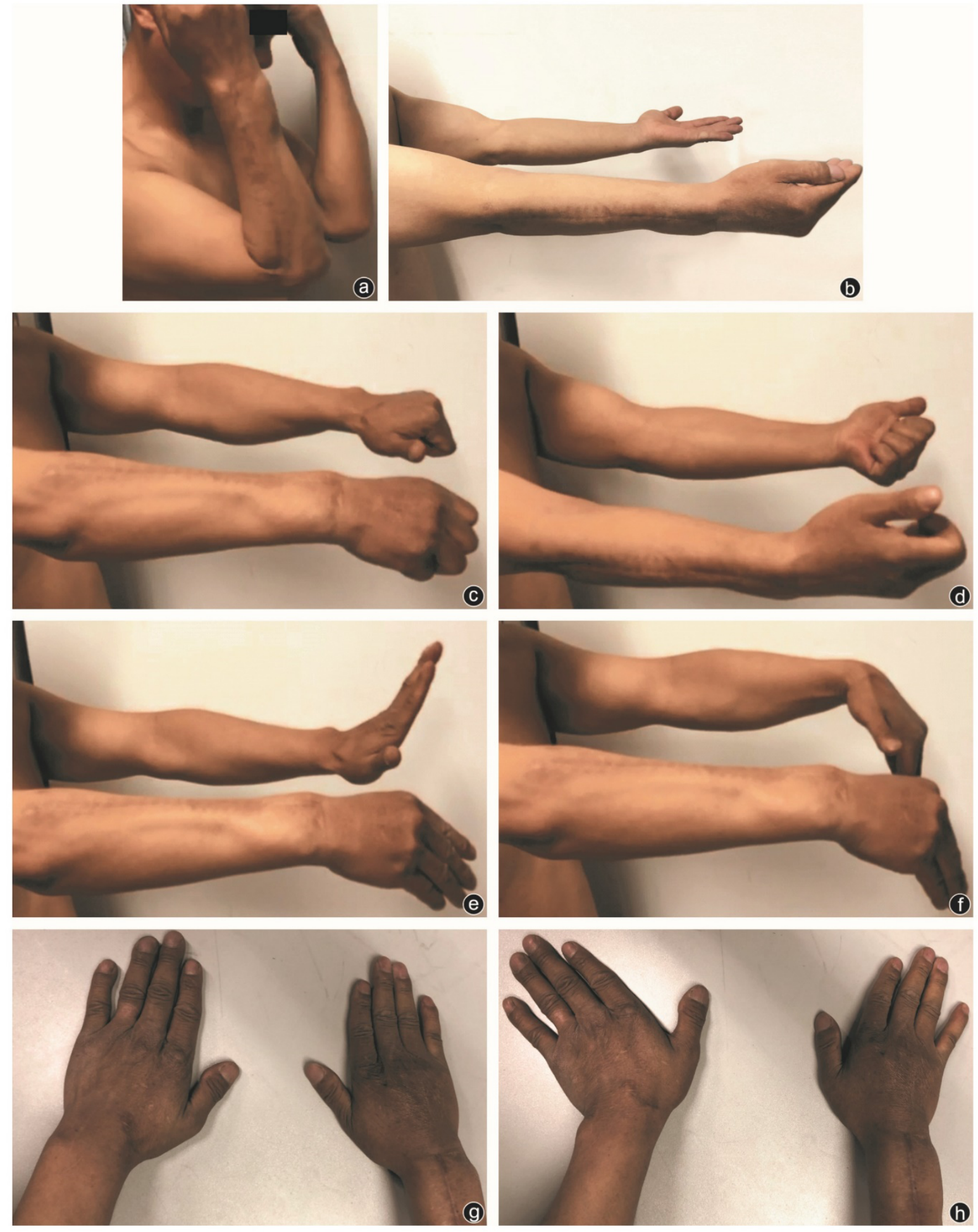

Orthopedic Science

Figure 4. 2-year follow-up of the forearm function test

a, b: Elbow joint mobility; c, d: forearm rotation function; $\mathrm{e}, \mathrm{h}$ : wrist joint mobility

nicely and the reconstructed bone healed up 3 months after surgery. The bone end fused completely 2 years after surgery (Figure 3). During the follow-up and 2 years after surgery, the appearance of the right forearm was normal, and there was no dislocation on the elbow and wrist joints. No tumor recurrence was observed. The forearm motor 
functions were elbow flexion $120^{\circ}$, straightening $0^{\circ}$, forearm anterior rotation $45^{\circ}$, posterior rotation $80^{\circ}$, the dorsal extension of wrist $10^{\circ}$, palm flexion $40^{\circ}$, wrist joint adduction $10^{\circ}$, and abduction $20^{\circ}$ (Figure 4). Elbow joint Mayo score was excellent, and the wrist joint Mayo scored well. Only forearm rotation and wrist extension and palm flexion were mildly limited that were not affected daily life.

\section{Discussion}

Chondrosarcoma is the formation of cartilage-like tissue by tumor cells, which can occur in any bone, but is commonly seen in long bones. The tumor reported in this article is extremely rare.

Malignant chondrosarcomas are pathologically classified as classical chondrosarcoma by pathology (grade I III). Low-grade malignant chondrosarcoma has a good overall prognosis but is highly invasive; chondrosarcoma has a high metastatic rate and is not sensitive to chemotherapy or radiotherapy [3]. Wide resection is the primary choice of treatment. If intraoperative is not possible to meet the boundary requirements for extended resection, it is often necessary to perform radiotherapy in the surgical field [4]. The patient's tumor pathology grade was III, and the clinical stage was II A (G1T1M0).

We performed extensive tumor resection plus postoperative radiotherapy and chemotherapy. No tumor recurrence was seen in 2 years follow-up, and the treatment outcome was good. After extensive resection of bone, the patient needed a lot of bone reconstruction, and the function of elbow and wrist joints should be guaranteed as much as possible. At present, the reconstruction method mainly uses its own body bone graft, allogeneic bone graft, and prosthesis replacement, but most of them are used for bone reconstruction after radial-distal tumor resection [5].

The Chondrosarcoma at the proximal midpoint of the radius is rare, and reconstruction methods are rarely reported. Song et al. reported the reconstruction method of one case of resection of the proximal radius giant cell tumor, in which polyethylene lining was used as the radial head prosthesis [6]. A pin was inserted into the radial bone marrow cavity as the prosthesis handle, and nylon mesh covered the surface to attach the biceps tendon.

Zhu et al. treated several patients with proximal radius bone tumor, the autogenous ilium was implanted in the bone defect after surgery, and elastic intramedullary nails were placed in the radial bone cavity [7]. Sakayama et al. used proximal radius total resection treatment of giant bone cell tumor of the proximal radius, bone cement-filled at the defect site and the elbow joint was constructed by floating comb bone [8].

All the above reconstruction plans have achieved some short-term results, but there is a lack of strong clinical evidence. In this case, the patient's tumor involved the proximal and middle section of the radius and nearly the whole length of the bone (only $3.6 \mathrm{~cm}$ was left in the distal radius after the resection of the tumor). As the radial bone defect was large, the prosthesis and bone cement filling could not be used for reconstruction. The ulna contributes the most to the elbow joint, while radial bone contributes to the wrist joint. In this case, we choose autologous ulnar osteotomy to reconstruct the forearm bone scaffold with the proximal ulna and distal residual radius, avoiding complex surgical procedures and utilizing the most reliable healing properties of autologous bone, which was the most economical choice in this case.

The patient was followed up two years after surgery. The patient's forearm appearance was excellent, the elbow joint flexion and extension were normal, and only the dorsal extension and abduction function of the wrist was slightly limited. However, the right hand functional impact was less affected, and the daily living ability was close to normal.

\section{Conclusion}

It is important to reconstruct the forearm support structure and function by fusion with the metallic implant of the proximal, middle ulna, and the distal radius. It was an excellent alternative treatment of such malignant chondrosarcoma grade III tumor.

\section{Ethical Considerations}

\section{Compliance with ethical guidelines}

The patient has given written consent to use his information. The Ethics Committee of Ningxia Medical University General Hospital permitted to use the patient's information and approved this manuscript for publication.

\section{Funding}

This research did not receive any specific grant from funding agencies in the public, commercial, or non-profit sectors.

\section{Authors' contributions}


Conceptualization and writing-original draft: Shi Jiandang, Sayed Abdulla Jami; Investigation: Sayed Abdulla Jami, Zhanwen Zhou, and Liu Chang Hao; Methodology, resources, and writing-review \& Editing: All authors; Supervision: Shi Jiandang.

\section{Conflict of interest}

The authors declared no conflict of interest.

\section{References}

[1] Leddy LR, Holmes RE. Chondrosarcoma of bone. Cancer treat res. 2014; 162:117-30. [DOI:10.1007/978-3-319-07323-1_6] [PMID]

[2] Lenze U, Pohlig F, Knebel C, Lenze F, Harrasser N, Muhlhofer $\mathrm{H}$ et al. [Autologous fibula transplantation for reconstruction of bone defects (German)]. Der Orthopade. 2017; 46(8):648-55. [DOI:10.1007/s00132-017-3442-2] [PMID]

[3] Shemesh SS, Acevedo-Nieves JD, Pretell-Mazzini J. Treatment strategies for central low-grade chondrosarcoma of long bones: A systematic review of the literature and meta-analysis. Musculoskeletal Surgery. 2018; 102(2):95-109. [DOI:10.1007/s12306-017-0507-7] [PMID]

[4] Riedel RF, Larrier N, Dodd L, Kirsch D, Martinez S, Brigman BE. The clinical management of chondrosarcoma. Curr Treat Options Oncol. 2009; 10(1-2):94-106. [DOI:10.1007/s11864-0090088-2] [PMID]

[5] Houdek MT, Wagner ER, Bishop AT, Shin AY, Rose PS, Sim FH et al. Complications and Long-Term Outcomes of Free Fibula Reconstruction following Resection of a Malignant Tumor in the Extremities. Plast Reconstr Surg. 2017; 139(2):510e9e. [DOI:10.1097/PRS.0000000000003004] [PMID]

[6] Song WS, Cho WH, Kong CB, Jeon DG. Composite reconstruction after proximal radial giant cell tumor resection. Arch Orthop Trauma Surg. 2011; 131(5):627-30. [DOI:10.1007/s00402-010-1180-6] [PMID]

[7] Zhu B, Yang J, Cheng D, Yin X, Yang Q. Reconstruct the proximal radius with iliac graft and elastic intramedullary nail fixation after tumor resection. World J Surg Oncol. 2016;14(1):210. [DOI:10.1186/s12957-016-0964-8] [PMID] [PMCID]

[8] Sakayama K, Sugawara Y, Kidani T, Miyawaki J, Yamamoto $\mathrm{H}$. Bipolar-type floating radial head prosthesis for the treatment of giant cell tumor of the proximal radius: A case report. J Shoulder Elbow Surg. 2006; 15(5):e9-12. [DOI:10.1016/j.jse.2005.06.001] [PMID] 
This Page Intentionally Left Blank 